\title{
Heterorhabditis safricana n. sp. (Rhabditida: Heterorhabditidae), a new entomopathogenic nematode from South Africa
}

\author{
Antoinette P. MALAN ${ }^{1, *}$, Khuong B. NGUYEN ${ }^{2}$, Jeanne Y. DE WAAL ${ }^{1}$ and Louwrence TIEDT ${ }^{3}$ \\ ${ }^{1}$ Department of Conservation Ecology and Entomology, Faculty of AgriSciences, University of Stellenbosch, \\ Private Bag X1, Matieland 7602, South Africa \\ ${ }^{2}$ Entomology and Nematology Department, University of Florida, Gainesville, FL 32601-0620, USA \\ ${ }^{3}$ Laboratory for Electron Microscopy, North-West University, Potchefstroom Campus, Private Bag X6001,
}

Potchefstroom 2520, South Africa

\begin{abstract}
Summary - During a survey for entomopathogenic nematodes in the Western Cape Province of South Africa, a new species in the genus Heterorhabditis was collected from a peach orchard. The nematode was trapped by the insect-baiting technique using last instar larvae of Galleria mellonella. The infective juvenile of the new species differs from the morphologically closest species, $H$. marelatus, in shorter body length 600 (550-676) vs 685 (588-700) $\mu \mathrm{m}$ and the shorter tail of 93 (86-108) vs 107 (99-117) $\mu \mathrm{m}$. It differs from all other species in the vulva pattern of hermaphroditic female. The genital papillae of the male $H$. safricana n. sp. are typical for species in the megidis-group (three papillae in terminal group). The average length of the gubernaculum is longer than that of all other species ( 24 vs 19-23 $\mu \mathrm{m}$ ) and the gubernaculum length as a percentage of spicule length (53.9) is less than that of H. mexicana (56), similar to that of $H$. floridensis (53.8), but larger than that of all others (51 or less). For molecular characterisation, the species closest to $H$. safricana

sp. is $H$. marelatus. The length of the ITS rDNA sequence of the new species is characterised by 995 base pairs, identical to that n.

of $H$. marelatus, but differs from this species by 25 aligned positions, seven of which are unambiguous autapomorphies. Phylogenetic trees show further evidence of a separate species status for $H$. safricana n. sp.
\end{abstract}

Keywords - description, ITS rDNA, molecular, morphology, morphometrics, phylogeny, SEM, systematics, taxonomy.

Entomopathogenic nematodes (EPN) of the families Steinernematidae and Heterorhabditidae occur naturally in soil and are parasites of a wide range of insects. The infective juvenile (IJ), a third-stage juvenile specially adapted for survival and location of a host in the soil, is the only free-living stage. The nematode infects the insect host mainly through natural openings, releases a symbiotically associated bacterium, and together they kill and utilise the insect host as a food source. The family Heterorhabditidae consists of only one genus, Heterorhabditis Poinar, 1976. Currently there are 12 recognised species in this genus (Nguyen, 2006; Nguyen et al., 2006a). It has been shown that Heterorhabditis can be used as an effective biological control agent against a wide range of soil-borne insect pests (Klein, 1990; Shapiro-Ilan et al., 2002).

The first occurrence of a Steinernema sp. in South Africa was reported in 1953. This nematode was col- lected from larvae, pupae and adults of the maize beetle, Heteronychus arator (Fabricius) $(=H$. sanctae-helenae Blanch.), in a maize field in Grahamstown, Eastern Cape Province (Harington, 1953). Several years later, a survey was undertaken in Kwazulu Natal to search for effective nematodes to control the sugarcane borer, Eldana saccharina Walker (Spaull, 1988, 1990, 1991). During these surveys, many isolates of Heterorhabditis and Steinernema were obtained but no species identifications were made. The first species of EPN reported from South Africa was H. bacteriophora Poinar, 1976 (Grenier et al., 1996) from the Western Cape Province. This species was identified using satellite DNA as a diagnostic probe.

In a survey conducted in 2003 to detect the occurrence of EPN throughout the Western Cape Province of South Africa, some isolates of Steinernema and several isolates of Heterorhabditis were obtained. Steinernema was rare, the only species found during the survey being new 
to science and subsequently described as S. khoisanae Nguyen, Malan \& Gozel, 2006. From several isolates of Heterorhabditis, H. bacteriophora was identified as the dominant species (Malan et al., 2006). Heterorhabditis zealandica Poinar, 1990 and a new species in the genus were also found during this survey. The new species is described here as Heterorhabditis safricana n. sp. using morphological characters, morphometrics and molecular data.

\section{Materials and methods}

\section{NEMATODE SOURCE}

Heterorhabditis safricana n. sp., isolate SF281, was collected from a soil sample taken from a peach orchard in the Western Cape Province, South Africa $\left(32^{\circ} 48^{\prime} .10 \mathrm{~S} /\right.$ $18^{\circ} 42^{\prime} .34 \mathrm{E}$ ). Larvae of Galleria mellonella (L.) (the greater wax moth) were used to trap the nematodes from the soil sample (Bedding \& Akhurst, 1975). Infective juveniles were maintained in the laboratory by recycling through G. mellonella larvae every 3 months and harvesting during the first week of emergence (Dutky et al., 1964). Most of the original IJ isolated from the soil, recycled once through $G$. mellonella, were still infective after 48 months of storage and were used throughout this study. The IJ were stored horizontally in $150 \mathrm{ml}$ filtered water at $14^{\circ} \mathrm{C}$ in $500 \mathrm{ml}$ culture flasks with vented lids and shaken weekly.

\section{MORPHOLOGICAL OBSERVATIONS}

For morphological studies, ten G. mellonella larvae were inoculated with $200 \mathrm{IJ}$ each in $8.5 \mathrm{~cm}$ diam. Petri dishes lined with moistened filter paper and kept in the dark at $25^{\circ} \mathrm{C}$. After 2 days, the larvae were washed free of surface nematodes and transferred to clean, moistened, filter paper. The first generation hermaphrodites were obtained by dissecting the insect cadavers in Ringer's solution 4-5 days after the larvae had died, and amphimictic males and females were obtained after 6-7 days. Infective juveniles were harvested within the first 2 days of emergence (Nguyen \& Smart, 1995a) from White traps (Woodring \& Kaya, 1988).

For light microscopy, 20 live specimens of the different stages (hermaphrodites, females, males, IJ) were examined. Additional specimens of the different life stages were killed and fixed in TAF $\left(80^{\circ} \mathrm{C}\right)$ (Courtney et al., 1955) for further observations. Type specimens were processed to glycerin (Seinhorst, 1959) and mounted with cover glass supports to avoid flattening.

For the morphology of the bursa, ten live males were transferred to a small drop of lactophenol containing $0.2 \%$ acid fuchsin. After $30 \mathrm{~min}$, they were individually transferred to a drop of lactophenol on a glass slide and the tail was removed by using the slanted sharp edge of a syringe needle. By using a cover slide, the tail was rotated to obtain a ventral view of the tail (Nguyen et al., 2006a). A total of 20 males was observed by using this method.

\section{SCANNING ELECTRON MICROSCOPY (SEM)}

Hermaphrodites, males, females and IJ were fixed in $3 \%$ formalin for 2 days, dehydrated in a graded ethanol series, critical point dried with liquid $\mathrm{CO}_{2}$, mounted on SEM stubs, and coated with gold (Nguyen \& Smart, 1995a). Spicules and gubernacula were prepared by leaving 30 males in water for 7 days as suggested by Nguyen and Smart (1995b).

\section{MOLECULAR CHARACTERISATION}

The Heterorhabditis species used in this study were: H. safricana n. sp. strain South Africa (SF281); H. amazonensis Andaló, Nguyen \& Moino, 2006; H. bacteriophora, strain G2; H. baujardi Phan, Subbotin, Nguyen \& Moens, 2003, strain Vietnam; H. downesi Stock, Griffin \& Burnell, 2002, strain K122; H. floridensis Nguyen, Gozel, Koppenhöfer \& Adams, 2006; H. indica Poinar, Karunakar \& David, 1992, strain India; H. marelatus Liu \& Berry, 1996, strain OH10; H. megidis Poinar, Jackson \& Klein, 1987, strain AGC; H. mexicana Nguyen, ShapiroIlan, Stuart, McCoy, James \& Adams, 2004, strain MX4; and $H$. zealandica, strain Florida. All of these sequences, except those of $H$. safricana, were from Nguyen et al. (2004, 2006a). All accession numbers are cited on the phylogenetic tree.

DNA extraction, PCR amplification, sequencing and multiple alignments were done as reported by Nguyen $e t$ al. (2004).

Phylogenetic analysis was done using PAUP* (Swofford, 2002). For the parsimony analysis, the shortest trees were obtained using heuristic search (stepwise addition as-is, branch swapping TBR). Gaps in the matrix were treated as either missing data or a fifth base. Caenorhabditis elegans was used as outgroup taxon and to root the tree. For ML analysis, models of sequence evolution were evaluated using Modeltest 3.07 (Posada \& Crandall, 1998). Modeltest utilises both likelihood ratio tests 
(hLRT) and Akaike (AIC) which favoured the HKY + G and TVM $+\mathrm{G}$ models with a 0.7564, 07954 gamma distribution shape parameter, respectively. The ML tree was inferred using a neighbour-joining starting tree, with heuristic searching of tree space by TBR branch swapping. Branch support was estimated by bootstrap analysis (1000 replicates for MP and 100 replicates for ML) using the same parameters as the original search. For further species delimitation, we traced the phylogenetic relationships amongst Heterorhabditis species and a map of ITS character states that could be polarised unambiguously (MacClade 4.05). This process shows the numbers of transition/transversion sites and autapomorphies (unique, derived characters) that, when fixed within and among lineages, indicate lineage independence.

\section{Heterorhabditis safricana* ${ }^{*}$. sp.} (Figs 1-4)

\section{MEASUREMENTS}

See Tables 1-3.

\section{DESCRIPTION}

\section{Male}

Body curved ventrally when killed with heat. Head truncate, sometimes slightly swollen. Labial papillae six, cephalic papillae not observed. Stoma with sclerotised rod or barrel-shaped cheilorhabdions; other rhabdions not distinguishable, forming a funnel-shaped structure posterior to cheilorhabdions. Pharynx with cylindrical corpus, metacorpus sometimes slightly enlarged. Nerve ring surrounding isthmus just anterior to basal bulb. Basal bulb with reduced valve. Cardia present, protruding into intestine. Excretory pore usually posterior to basal bulb. Testis monorchic, reflexed. Vas deferens well developed. Spicules paired. Bursa peloderan. Genital papillae normally comprising nine pairs and typical for genus. From anterior to posterior, pair 1 well anterior to cloaca, pairs 2 and 3 forming a group, pairs 4, 5 and 6 forming a group with anterior pair in group curved laterally and slightly separated from posterior two, terminal group formed by pairs 7, 8 and 9, with pair 7 curved laterally. In general, anterior two pairs of papillae in terminal group weaker and shorter. Tail conoid, slightly curved ventrally.

\footnotetext{
* The specific epithet is derived from South Africa, the country of origin.
}

\section{Hermaphroditic female}

C-shaped after killing with gentle heat. Body robust, always with many eggs in young females and many eggs and juveniles in mature females. Cuticle smooth under light microscope but finely annulated under SEM. Head region tapering anteriorly. Labial region with six prominent lips. Amphidial apertures pore-like. Stoma with refractile cheilorhabdions. Posterior part of stoma funnel-shaped, enclosed by anterior part of pharynx. Pharynx with cylindrical corpus. Nerve ring in vicinity of narrowed isthmus between corpus and basal bulb. Basal bulb prominent with conspicuous valve, lumen of pharynx in basal bulb well sclerotised. Opening of excretory pore located posterior to end of pharynx. Cardia present, well developed. Gonads didelphic, amphidelphic. Vulva in form of a transverse slit, located anterior to midbody on slightly protruding area. In ventral view, vulva elliptical, encircled by arch-shaped annules anteriorly and posteriorly. Vagina short. Tail longer than anal body diam., conoid with pointed terminus. Near terminus, tail widening then narrowing to a pointed tip. Post-anal swelling present. Phasmid inconspicuous.

\section{Amphimictic female}

Similar to hermaphroditic female but smaller. Vulva not protruding, usually (i.e., in $60 \%$ of specimens) covered with exudates or copulation plug after mating. Post-anal swelling much smaller.

\section{Infective juvenile (IJ)}

Body elongate. Sheath (second-stage cuticle) present immediately after harvesting, but lost in storage. Labial region with six annules lacking longitudinal incisures. Anterior part of body of ensheathed juvenile with tessellate pattern, posterior part with longitudinal ridges. Tail long, pointed. Exsheathed IJ body annulated, longitudinal ridges absent. Amphidial apertures prominent, pore-like. Excretory duct pronounced, well cuticularised (except in anterior part) 3-5 $\mu \mathrm{m}$ from excretory pore. Pharynx typical of genus. Lateral field at mid-body with two ridges. Phasmid not observed under light microscope. Tail elongate conoid with pointed terminus. Tail length without sheath $c a 61 \%$ of tail with sheath.

\section{TYPE HOST AND LOCALITY}

The natural host of $H$. safricana $\mathrm{n}$. sp. is unknown as the nematode was trapped by baiting with $G$. mellonella. Soil samples were obtained from a peach orchard on Hochland 

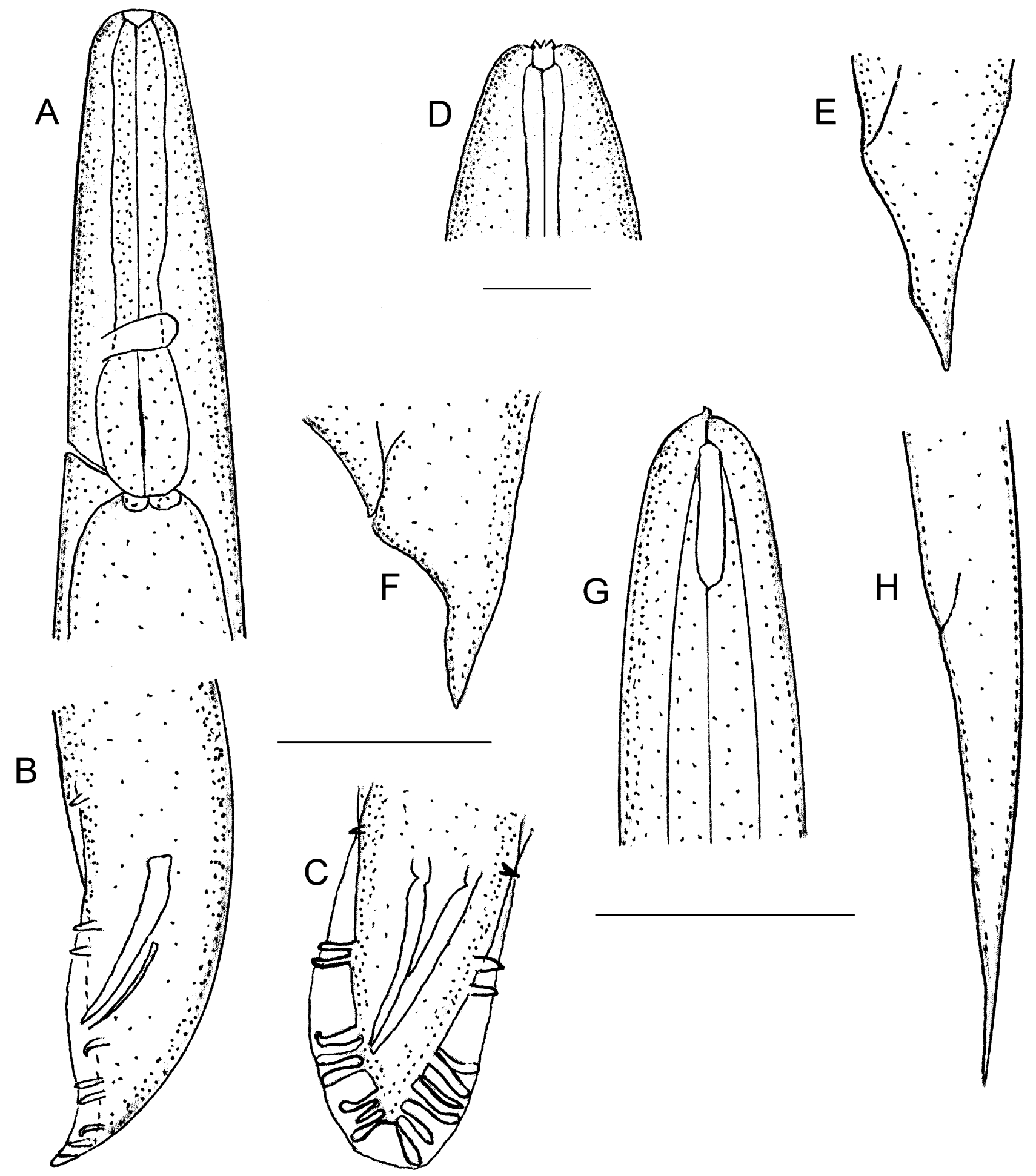

Fig. 1. Heterorhabditis safricana $n . s p$. A-C: Male. A: Anterior region; B, C: Lateral and ventral view of tail showing spicules, gubernaculum and genital papillae. D-F: Female head and variation of tail. G, H: Infective juvenile. G: Anterior region; H: Posterior region. (Scale bars: $A-C, E, F, H$ (left bar $)=50 \mu \mathrm{m} ; D$ (top bar $)=50 \mu \mathrm{m} ; G$ (right bar $)=25 \mu \mathrm{m}$.) 
Table 1. Morphometrics of Heterorhabditis safricana $n$. sp. All measurements in $\mu m$ and in the form: mean \pm sd (range).

\begin{tabular}{|c|c|c|c|c|c|}
\hline \multirow[t]{2}{*}{ Parameter } & \multicolumn{2}{|c|}{ Male } & \multirow{2}{*}{$\begin{array}{l}\text { Hermaphrodite } \\
\text { (1st generation) }\end{array}$} & \multirow{2}{*}{$\begin{array}{c}\text { Female } \\
\frac{(2 n d \text { generation })}{\text { Paratypes }}\end{array}$} & \multirow{2}{*}{$\begin{array}{c}\begin{array}{l}\text { Infective } \\
\text { juvenile }\end{array} \\
\text { Paratypes }\end{array}$} \\
\hline & Holotype & Paratypes & & & \\
\hline $\mathrm{n}$ & - & 20 & 20 & 20 & 25 \\
\hline $\mathrm{L}$ & 946 & $\begin{array}{c}892 \pm 66 \\
(777-1009)\end{array}$ & $\begin{array}{c}3713 \pm 203 \\
(3373-4073)\end{array}$ & $\begin{array}{c}2292 \pm 302 \\
(1679-2937)\end{array}$ & $\begin{array}{l}600 \pm 27 \\
(550-676)\end{array}$ \\
\hline $\mathrm{a}$ & - & - & - & - & $\begin{array}{l}28.6 \pm 1.7 \\
(24.8-31.8)\end{array}$ \\
\hline $\mathrm{b}$ & - & - & - & - & $\begin{array}{l}4.5 \pm 0.2 \\
(3.9-4.9)\end{array}$ \\
\hline $\mathrm{c}$ & - & - & - & - & $\begin{array}{l}6.4 \pm 0.6 \\
(5.4-7.5)\end{array}$ \\
\hline V & - & - & $\begin{array}{c}44 \pm 0.8 \\
(43-46)\end{array}$ & $\begin{array}{c}48 \pm 1.2 \\
(45-50)\end{array}$ & - \\
\hline Max body diam. & 47 & $\begin{array}{c}49 \pm 4.5 \\
(40-58)\end{array}$ & $\begin{array}{l}155 \pm 16 \\
(127-188)\end{array}$ & $\begin{array}{c}171 \pm 28.8 \\
(102-229)\end{array}$ & $\begin{array}{c}21 \pm 1.2 \\
(19-23)\end{array}$ \\
\hline Stoma length & 8.0 & $\begin{array}{l}8.3 \pm 1.0 \\
(6.3-9.8)\end{array}$ & $\begin{array}{l}13.5 \pm 2.4 \\
(10.0-18.6)\end{array}$ & $\begin{array}{l}11.2 \pm 1.3 \\
(8.5-12.7)\end{array}$ & - \\
\hline Stoma diam. & 5.4 & $\begin{array}{l}5.8 \pm 1.1 \\
(4.5-8.9)\end{array}$ & $\begin{array}{l}10.4 \pm 1.7 \\
(8.6-14.3)\end{array}$ & $\begin{array}{l}9.4 \pm 1.5 \\
(7.0-12.7)\end{array}$ & - \\
\hline $\mathrm{EP}$ & 145 & $\begin{array}{c}135 \pm 11 \\
(104-147)\end{array}$ & $\begin{array}{l}230 \pm 14 \\
(210-267)\end{array}$ & $\begin{array}{c}171 \pm 11 \\
(151-196)\end{array}$ & $\begin{array}{l}110 \pm 4.2 \\
(103-122)\end{array}$ \\
\hline NR & 66 & $\begin{array}{l}64 \pm 8 \\
(52-81)\end{array}$ & $\begin{array}{c}139 \pm 12 \\
(121-163)\end{array}$ & $\begin{array}{l}111 \pm 11 \\
(87-139)\end{array}$ & $\begin{array}{l}93 \pm 4.0 \\
(86-101)\end{array}$ \\
\hline ES & 119 & $\begin{array}{c}115 \pm 5 \\
(105-126)\end{array}$ & $\begin{array}{c}215 \pm 9 \\
(199-236)\end{array}$ & $\begin{array}{c}168 \pm 8 \\
(148-180)\end{array}$ & $\begin{array}{l}131 \pm 3.7 \\
(125-141)\end{array}$ \\
\hline Testis reflexion & 88 & $\begin{array}{l}93 \pm 22 \\
(52-129)\end{array}$ & - & - & - \\
\hline Tail length with sheath $(\mathrm{T})$ & - & - & - & - & $\begin{array}{l}93 \pm 5.5 \\
(86-108)\end{array}$ \\
\hline Tail length without sheath & 33 & $\begin{array}{l}35 \pm 5 \\
(27-49)\end{array}$ & $\begin{array}{c}77 \pm 8 \\
(64-91)\end{array}$ & $\begin{array}{l}69 \pm 14 \\
(55-111)\end{array}$ & $\begin{array}{c}57 \pm 3.0 \\
(49-62)\end{array}$ \\
\hline Anal body diam. (ABD) & 22 & $\begin{array}{l}24 \pm 3 \\
(18-27)\end{array}$ & $\begin{array}{l}46 \pm 5 \\
(40-54)\end{array}$ & $\begin{array}{l}35 \pm 11 \\
(25-72)\end{array}$ & $\begin{array}{l}13 \pm 0.6 \\
(12-14)\end{array}$ \\
\hline Spicule length (SP) & 47 & $\begin{array}{l}45 \pm 4 \\
(35-54)\end{array}$ & - & - & - \\
\hline Spicule width & 7.1 & $\begin{array}{l}5.7 \pm 1.1 \\
(3.6-7.1)\end{array}$ & - & - & - \\
\hline Gubernaculum length (GU) & 26 & $\begin{array}{c}24 \pm 2 \\
(19-27)\end{array}$ & - & - & - \\
\hline $\mathrm{D}(\%)=(\mathrm{EP} / \mathrm{ES}) \times 100$ & 122 & $\begin{array}{l}117 \pm 10 \\
(92-133)\end{array}$ & $\begin{array}{l}107 \pm 6 \\
(98-119)\end{array}$ & $\begin{array}{l}108 \pm 6 \\
(97-120)\end{array}$ & $\begin{array}{c}84 \pm 2.6 \\
(80-90)\end{array}$ \\
\hline $\mathrm{E}(\%)=(\mathrm{EP} / \mathrm{T}) \times 100$ & - & - & - & - & $\begin{array}{l}119 \pm 9 \\
(99-133)\end{array}$ \\
\hline $\mathrm{SW} \%=\mathrm{SP} / \mathrm{ABD} \times 100$ & 212 & $\begin{array}{l}196 \pm 32 \\
(130-259)\end{array}$ & - & - & - \\
\hline $\mathrm{GS} \%=\mathrm{GU} / \mathrm{SP} \times 100$ & 55 & $\begin{array}{l}53.9 \pm 4.7 \\
(43.3-61.7)\end{array}$ & - & - & - \\
\hline
\end{tabular}

$\mathrm{EP}=$ distance from anterior end to excretory pore; $\mathrm{NR}=$ distance from anterior end to nerve ring; $\mathrm{ES}=$ distance from anterior end to end of pharynx. 

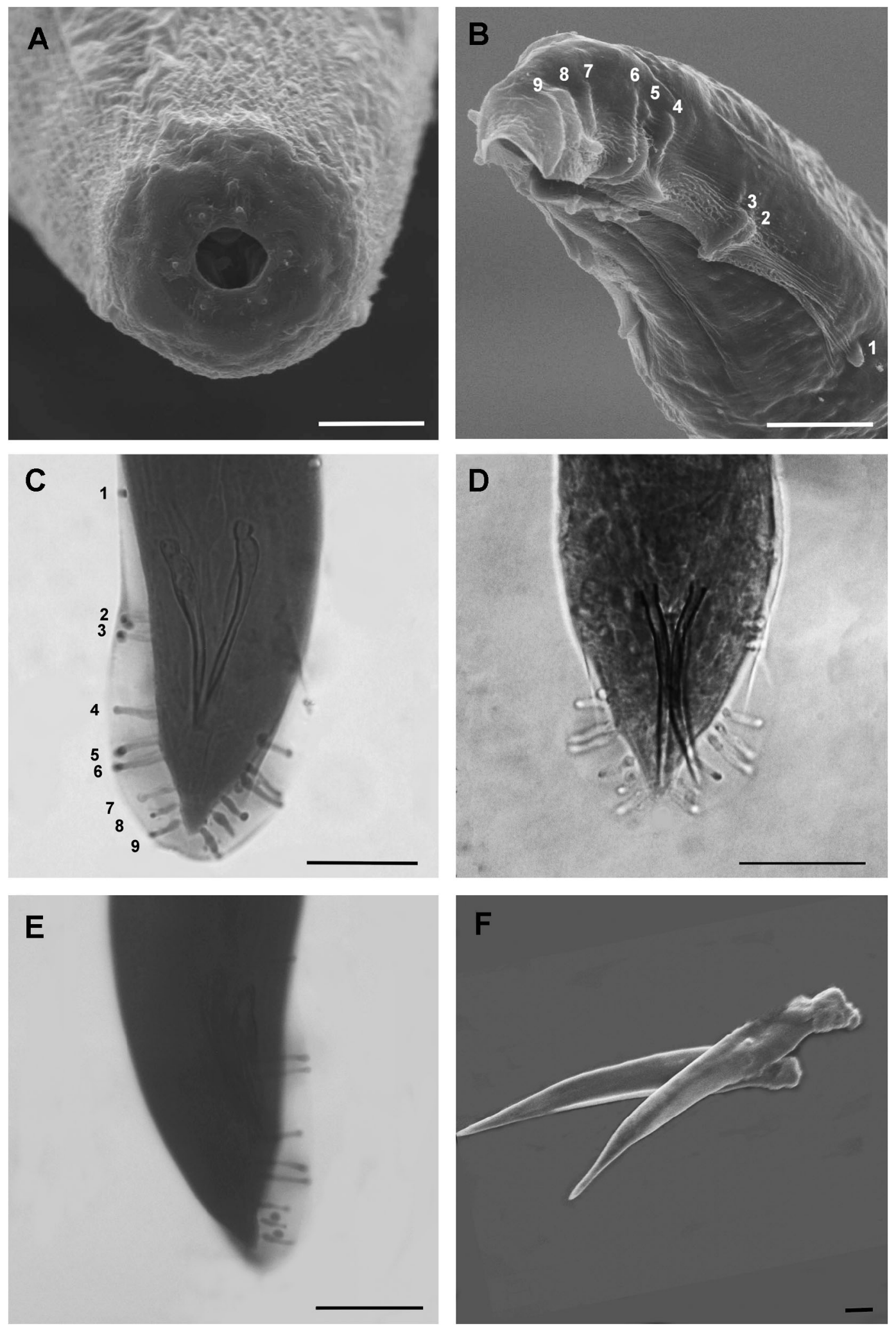

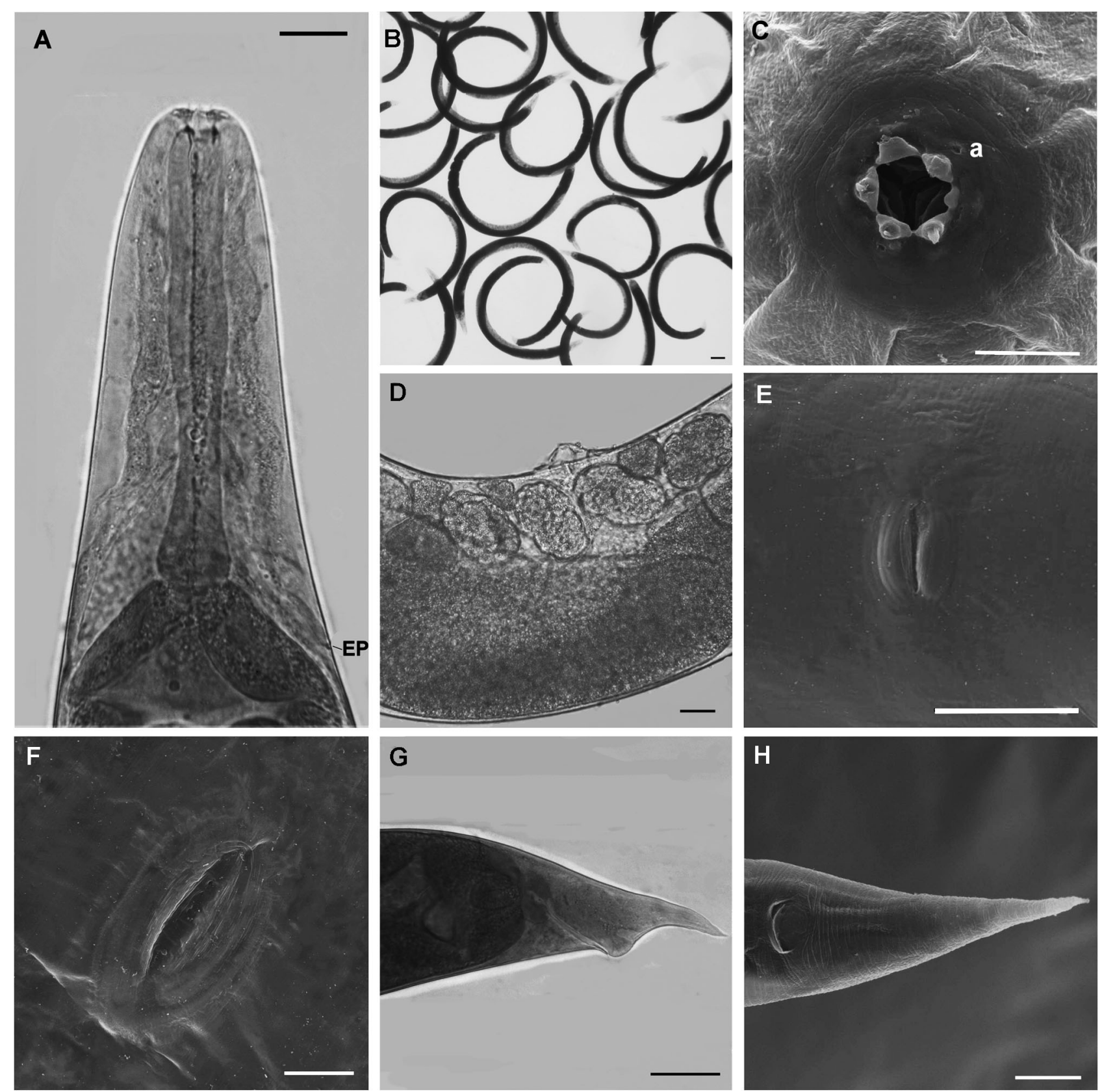

Fig. 3. Heterorhabditis safricana $n$. sp. A, B, D, G: Light microscope photographs; $C, E, F, H$ : SEM photographs. A: Pharynx of hermaphroditic female showing position of excretory pore (EP); B: C-shaped body of hermaphroditic females; $C$ : En face view of amphimictic female showing six prominent lips, labial papillae and amphidial apertures (a); D: Vulva of amphimictic female covered with exudates or copulation plug; E, F: Vulva with elliptical pattern; G: Hermaphroditic female tail, lateral view; H: Hermaphroditic female tail, ventral view. (Scale bars: $A, D, E, G=20 \mu \mathrm{m} ; C, F, H=10 \mu \mathrm{m} ; B=150 \mu \mathrm{m}$.)

Fig. 2. Heterorhabditis safricana n. sp. A, B, F: SEM photographs of male; C-E: Light microscope photographs. A: Head region showing mouth opening and six labial papillae; B: Posterior region showing anal aperture and bursa with full complement of papillae; $C$, D: Male tails with bursal papillae; E: Lateral view of male tail with bursa and papillae; F: Spicules. (Scale bars: $A=5 \mu m ; B=20 \mu m$; $C-E=24 \mu m ; F=3 \mu m$.) 

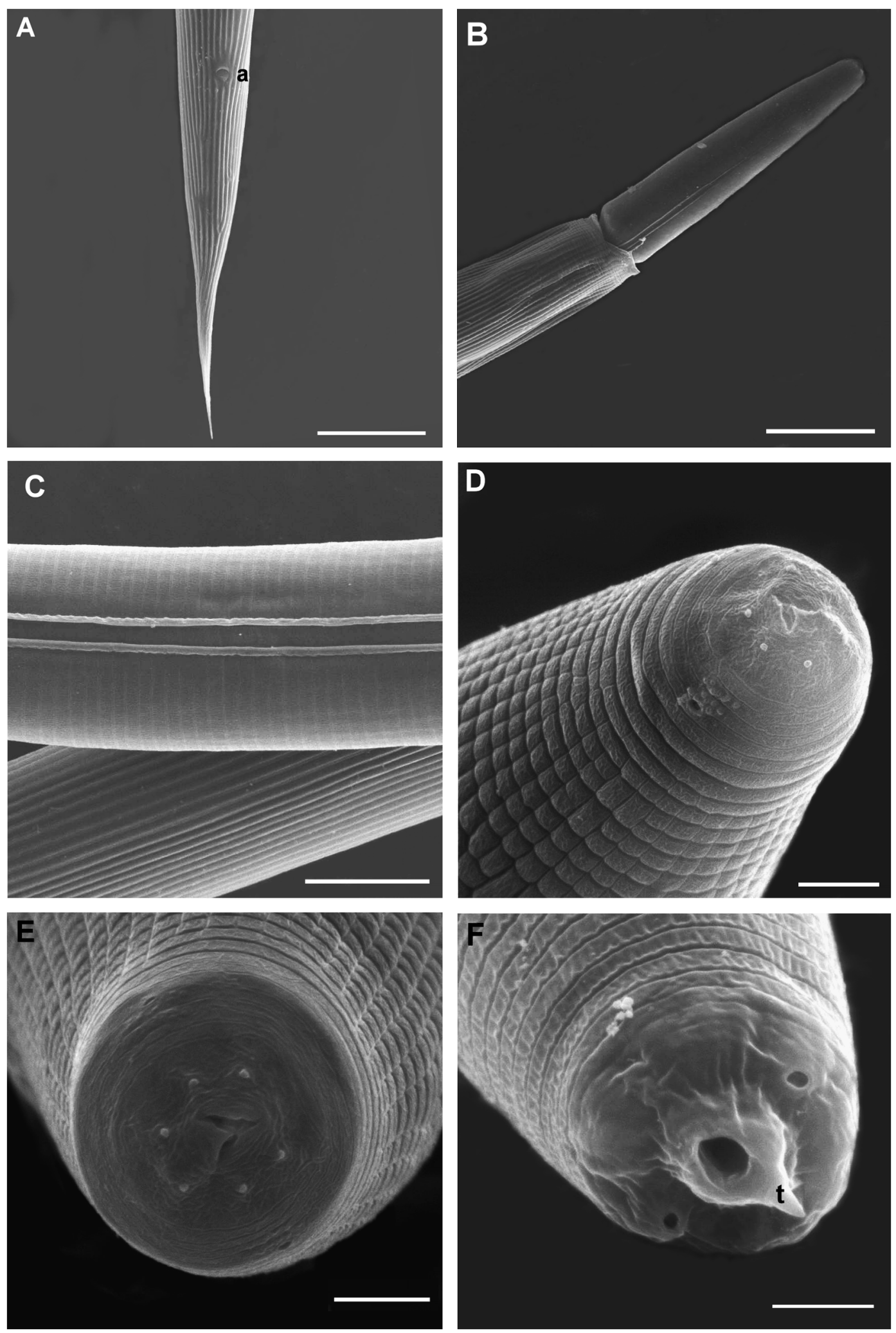
Table 2. Comparative morphometrics of male of Heterorhabditis safricana $n$. sp. and other related species. All measurements in $\mu m$ and in the form: mean (range) or mean \pm s.d. (range).

\begin{tabular}{|c|c|c|c|c|c|c|}
\hline Parameter & $\begin{array}{c}\text { MAR } \\
\text { (after Liu \& } \\
\text { Berry, 1996) }\end{array}$ & $\begin{array}{c}\text { ZEA } \\
\text { (after Nguyen } \\
\text { et al., 2005) }\end{array}$ & $\begin{array}{c}\text { SAF } \\
\text { (present } \\
\text { study) }\end{array}$ & $\begin{array}{c}\text { BAC } \\
\text { (after Poinar, } \\
1990)\end{array}$ & $\begin{array}{c}\text { DOW } \\
\text { (after Stock } \\
\text { et al., 2002) }\end{array}$ & $\begin{array}{c}\text { MEG } \\
\text { (after Poinar } \\
\text { et al., 1987) }\end{array}$ \\
\hline $\mathrm{n}$ & 20 & 15 & 20 & 15 & 19 & 15 \\
\hline $\mathrm{L}$ & $\begin{array}{c}942 \pm 77 \\
(805-1046)\end{array}$ & $\begin{array}{c}914 \\
(848-1044)\end{array}$ & $\begin{array}{c}892 \pm 66 \\
(777-1009)\end{array}$ & $\begin{array}{c}820 \\
(780-960)\end{array}$ & $\begin{array}{l}800 \pm 76 \\
(669-876)\end{array}$ & $\begin{array}{c}1000 \\
(800-1100)\end{array}$ \\
\hline Max. body diam. & $\begin{array}{l}51 \pm 4 \\
(48-56)\end{array}$ & $\begin{array}{c}41 \\
(36-45)\end{array}$ & $\begin{array}{c}49 \pm 4.5 \\
(40-58)\end{array}$ & $\begin{array}{c}43 \\
(38-46)\end{array}$ & $\begin{array}{l}36 \pm 3 \\
(33-40)\end{array}$ & $\begin{array}{c}47 \\
(44-50)\end{array}$ \\
\hline $\mathrm{EP}$ & $\begin{array}{l}130 \pm 30 \\
(111-168)\end{array}$ & $\begin{array}{c}139 \\
(130-150)\end{array}$ & $\begin{array}{c}135 \pm 11 \\
(104-147)\end{array}$ & $\begin{array}{c}121 \\
(114-130)\end{array}$ & $\begin{array}{l}89 \pm 2 \\
(86-91)\end{array}$ & $\begin{array}{c}156 \\
(139-176)\end{array}$ \\
\hline NR & $\begin{array}{l}73 \pm 9 \\
(61-95)\end{array}$ & - & $\begin{array}{l}64 \pm 8 \\
(52-81)\end{array}$ & $\begin{array}{c}72 \\
(65-81)\end{array}$ & $\begin{array}{l}70 \pm 7 \\
(86-91)\end{array}$ & $\begin{array}{c}104 \\
(96-112)\end{array}$ \\
\hline ES & $\begin{array}{l}115 \pm 6 \\
(99-123)\end{array}$ & $\begin{array}{c}118 \\
(110-128)\end{array}$ & $\begin{array}{c}115 \pm 5 \\
(105-126)\end{array}$ & $\begin{array}{c}103 \\
(99-105)\end{array}$ & $\begin{array}{l}101 \pm 3 \\
(97-106)\end{array}$ & $\begin{array}{c}128 \\
(122-134)\end{array}$ \\
\hline Tail length & $\begin{array}{l}31 \pm 3 \\
(34-38)\end{array}$ & $\begin{array}{c}35 \\
(30-41)\end{array}$ & $\begin{array}{l}35 \pm 5 \\
(27-49)\end{array}$ & $\begin{array}{c}28 \\
(22-36)\end{array}$ & $\begin{array}{l}32 \pm 2 \\
(29-34)\end{array}$ & $\begin{array}{c}39 \\
(35-43)\end{array}$ \\
\hline Anal body diam. (ABD) & $\begin{array}{l}23 \pm 2 \\
(20-28)\end{array}$ & $\begin{array}{c}20.7 \\
(18-24)\end{array}$ & $\begin{array}{l}24 \pm 3 \\
(18-27)\end{array}$ & $\begin{array}{c}23 \\
(22-25)\end{array}$ & $\begin{array}{l}24 \pm 2 \\
(21-28)\end{array}$ & $\begin{array}{c}26 \\
(22-31)\end{array}$ \\
\hline Spicule length (SP) & $\begin{array}{l}45 \pm 3 \\
(42-50)\end{array}$ & $\begin{array}{c}51 \\
(48-55)\end{array}$ & $\begin{array}{l}45 \pm 4 \\
(35-54)\end{array}$ & $\begin{array}{c}40 \\
(36-44)\end{array}$ & $\begin{array}{l}43 \pm 2 \\
(41-47)\end{array}$ & $\begin{array}{c}49 \\
(46-54)\end{array}$ \\
\hline Gubernaculum length (GU) & $\begin{array}{c}19 \pm 1 \\
(18-22)\end{array}$ & $\begin{array}{c}22 \\
(19-25)\end{array}$ & $\begin{array}{c}24 \pm 2 \\
(19-27)\end{array}$ & $\begin{array}{c}20 \\
(18-25)\end{array}$ & $\begin{array}{c}18 \pm 1 \\
(17-20)\end{array}$ & $\begin{array}{c}21 \\
(17-24)\end{array}$ \\
\hline $\mathrm{D}(\%)=(\mathrm{EP} / \mathrm{ES}) \times 100$ & 113 & 118 & $\begin{array}{l}117 \pm 10 \\
(92-133)\end{array}$ & 117 & 88 & 122 \\
\hline $\mathrm{SW}(\%)=(\mathrm{SP} / \mathrm{ABW}) \times 100$ & $\begin{array}{c}175 \\
(173-178)\end{array}$ & $\begin{array}{c}181 \\
(160-209)\end{array}$ & $\begin{array}{c}196 \pm 32 \\
(130-259)\end{array}$ & 174 & $\begin{array}{c}180 \pm 20 \\
(170-220)\end{array}$ & 188 \\
\hline $\mathrm{GS}(\%)=(\mathrm{GU} / \mathrm{SP}) \times 100$ & $\begin{array}{l}41 \pm 4 \\
(36-50)\end{array}$ & 43 & $\begin{array}{l}54 \pm 5 \\
(43-62)\end{array}$ & 50 & $\begin{array}{l}43 \pm 4 \\
(36-47)\end{array}$ & 43 \\
\hline
\end{tabular}

$\mathrm{EP}=$ distance from anterior end to excretory pore; $\mathrm{NR}=$ distance from anterior end to nerve ring; $\mathrm{ES}=$ distance from anterior end to end of pharynx; $\mathrm{BAC}=H$. bacteriophora $\mathrm{SAF}=H$. safricana $\mathrm{n} . \mathrm{sp} . ; \mathrm{DOW}=H$. downesi , MAR $=H$. marelatus; $\mathrm{ZEA}=H$. zealandica $\mathrm{MEG}=$ H. megidis.

farm in the Western Cape Province $\left(32^{\circ} 48^{\prime} .10 \mathrm{~S} / 18^{\circ} 42^{\prime}\right.$. 34E), South Africa.

\section{TYPE MATERIAL}

Holotype male (permanent mount in glycerin) and 20 paratypes (all in TAF) of each of hermaphrodite, female and male, together with many IJ, isolated from the haemocoel of G. mellonella, deposited in the United States
Department of Agriculture Nematode Collection (USDANC), Beltsville, MD, USA. In addition, 20 paratypes (all in TAF) of each of hermaphrodite, male, female, together with many IJ, deposited in the National Collection of Nematodes, Biosystematics Division, Plant Protection Research Institute, Agricultural Research Council, Pretoria, South Africa. Slides of one paratype male and one paratype female of the second generation, together with several IJ, deposited in the California Collection of Ne-

Fig. 4. Heterorhabditis safricana n. sp. A-F: SEM photographs of infective juvenile (IJ). A: Tail of ensheathed IJ showing anus (a) and longitudinal ridges; B: Anterior end of a partially sheathed IJ; C: Longitudinal ridges of ensheathed IJ (bottom nematode) and lateral field at mid-body with two ridges of exsheathed IJ (top nematode); D: Anterior end of ensheathed IJ showing tessellate pattern, labial papillae, mouth opening and amphidial aperture; E: Anterior end of ensheathed IJ showing six labial papillae; F: Exsheathed IJ showing dorsal tooth ( $t$ ). (Scale bars: $A, B=20 \mu \mathrm{m} ; C-F=2 \mu \mathrm{m}$. ) 
Table 3. Comparative morphometrics of infective juveniles of Heterorhabditis safricana $n$. sp. and other related species. All measurements in $\mu \mathrm{m}$ and in the form: mean (range) or mean \pm s.d. (range).

\begin{tabular}{|c|c|c|c|c|c|c|}
\hline Parameter & $\begin{array}{c}\text { BAC } \\
\text { (after Poinar, } \\
1990)\end{array}$ & $\begin{array}{c}\text { SAF } \\
\text { (present } \\
\text { study) }\end{array}$ & $\begin{array}{c}\text { DOW } \\
\text { (after Stock } \\
\text { et al., 2002) }\end{array}$ & $\begin{array}{c}\text { MAR } \\
\text { (after Liu \& } \\
\text { Berry, 1996) }\end{array}$ & $\begin{array}{c}\text { ZEA } \\
\text { (after Nguyen } \\
\text { et al., 2005) }\end{array}$ & $\begin{array}{c}\text { MEG } \\
\text { (after Poinar } \\
\text { et al., 1987) }\end{array}$ \\
\hline $\mathrm{n}$ & 25 & 25 & 20 & 20 & 25 & 15 \\
\hline $\mathrm{L}$ & $\begin{array}{c}588 \\
(512-671)\end{array}$ & $\begin{array}{l}600 \pm 27 \\
(550-676)\end{array}$ & $\begin{array}{l}637 \pm 32 \\
(588-692)\end{array}$ & $\begin{array}{l}654 \pm 26 \\
(588-700)\end{array}$ & $\begin{array}{c}685 \\
(570-740)\end{array}$ & $\begin{array}{c}768 \\
(736-800)\end{array}$ \\
\hline $\mathrm{a}$ & $\begin{array}{c}25 \\
(17-30)\end{array}$ & $\begin{array}{l}28.6 \pm 1.7 \\
(24.8-31.8)\end{array}$ & $\begin{array}{c}35 \pm 4 \\
(4.4-5.3)\end{array}$ & $\begin{array}{l}23.9 \pm 2.5 \\
(20.5-29.0)\end{array}$ & - & $\begin{array}{c}26 \\
(23-28)\end{array}$ \\
\hline $\mathrm{b}$ & $\begin{array}{c}4.5 \\
(4.0-5.1)\end{array}$ & $\begin{array}{l}4.5 \pm 0.2 \\
(3.9-4.9)\end{array}$ & $\begin{array}{l}4.7 \pm 0.3 \\
(4.4-5.3)\end{array}$ & $\begin{array}{c}4.9 \pm 0.2 \\
(4.7-5.4)\end{array}$ & - & $\begin{array}{c}5.0 \\
(4.6-5.0)\end{array}$ \\
\hline $\mathrm{c}$ & $\begin{array}{c}6.2 \\
(5.5-7.0)\end{array}$ & $\begin{array}{l}6.4 \pm 0.6 \\
(5.4-7.5)\end{array}$ & $\begin{array}{l}9.5 \pm 0.5 \\
(8.5-10.5)\end{array}$ & $\begin{array}{l}6.1 \pm 0.3 \\
(5.5-6.6)\end{array}$ & $\begin{array}{c}6.6 \\
(6.2-6.7)\end{array}$ & $\begin{array}{c}6.5 \\
(6.1-6.9)\end{array}$ \\
\hline Max. body diam. & $\begin{array}{c}23 \\
(18-31)\end{array}$ & $\begin{array}{c}21 \pm 1.2 \\
(18-23)\end{array}$ & $\begin{array}{l}18 \pm 2 \\
(15-22)\end{array}$ & $\begin{array}{l}28 \pm 3 \\
(24-32)\end{array}$ & $\begin{array}{c}27 \\
(22-30)\end{array}$ & $\begin{array}{c}29 \\
(27-32)\end{array}$ \\
\hline $\mathrm{EP}$ & $\begin{array}{c}103 \\
(87-110)\end{array}$ & $\begin{array}{l}110 \pm 4.2 \\
(103-122)\end{array}$ & $\begin{array}{l}115 \pm 8 \\
(96-128)\end{array}$ & $\begin{array}{l}102 \pm 7 \\
(81-113)\end{array}$ & $\begin{array}{c}140 \\
(135-147)\end{array}$ & $\begin{array}{c}131 \\
(123-142)\end{array}$ \\
\hline NR & $\begin{array}{c}85 \\
(72-93)\end{array}$ & $\begin{array}{l}93 \pm 4.0 \\
(86-101)\end{array}$ & $\begin{array}{l}101 \pm 3 \\
(96-105)\end{array}$ & $\begin{array}{c}98.5 \pm 8.8 \\
(83.2-112.9)\end{array}$ & $\begin{array}{c}100 \\
(90-107)\end{array}$ & $\begin{array}{c}131 \\
(123-142)\end{array}$ \\
\hline ES & $\begin{array}{c}125 \\
(100-139)\end{array}$ & $\begin{array}{l}131 \pm 3.7 \\
(125-141)\end{array}$ & $\begin{array}{c}135 \pm 4 \\
(126-141)\end{array}$ & $\begin{array}{c}132.7 \pm 4.8 \\
(121-139)\end{array}$ & - & $\begin{array}{c}155 \\
(147-160)\end{array}$ \\
\hline $\begin{array}{l}\text { Tail length with sheath } \\
\text { (T) }\end{array}$ & $\begin{array}{c}98 \\
(83-112)\end{array}$ & $\begin{array}{l}93 \pm 5.5 \\
(86-108)\end{array}$ & $\begin{array}{l}68 \pm 4 \\
(62-74)\end{array}$ & $\begin{array}{l}107 \pm 5 \\
(99-117)\end{array}$ & $\begin{array}{c}102 \\
(87-119)\end{array}$ & $\begin{array}{c}119 \\
(112-128)\end{array}$ \\
\hline $\begin{array}{l}\text { Tail length without } \\
\text { sheath }\end{array}$ & - & $\begin{array}{l}57 \pm 3.0 \\
(49-62)\end{array}$ & - & - & - & - \\
\hline Anal body diam. & - & $\begin{array}{c}13 \pm 0.6 \\
(12-14)\end{array}$ & $\begin{array}{l}12 \pm 1 \\
(9-14)\end{array}$ & - & - & - \\
\hline $\mathrm{D}(\%)=(\mathrm{EP} / \mathrm{ES}) \times 100$ & $\begin{array}{c}84 \\
(76-92)\end{array}$ & $\begin{array}{c}84 \pm 2.6 \\
(80-90)\end{array}$ & $\begin{array}{l}85 \pm 5 \\
(76-98)\end{array}$ & $\begin{array}{c}77 \pm 0.1 \\
(60-86)\end{array}$ & - & - \\
\hline $\mathrm{E}(\%)=(\mathrm{EP} / \mathrm{T}) \times 100$ & $\begin{array}{c}112 \\
(103-130)\end{array}$ & $\begin{array}{l}119 \pm 9 \\
(99-133)\end{array}$ & $\begin{array}{l}170 \pm 10 \\
(160-180)\end{array}$ & $\begin{array}{c}96 \pm 8 \\
(89-110)\end{array}$ & $\begin{array}{c}108 \\
(103-109)\end{array}$ & $\begin{array}{c}110 \\
(103-120)\end{array}$ \\
\hline
\end{tabular}

$\mathrm{EP}=$ distance from anterior end to excretory pore; $\mathrm{NR}=$ distance from anterior end to nerve ring; $\mathrm{ES}=$ distance from anterior end to end of pharynx; $\mathrm{BAC}=H$. bacteriophora; $\mathrm{SAF}=H$. safricana $\mathrm{n}$. sp.; DOW $=H$. downesi $\mathrm{MAR}=H$. marelatus; $\mathrm{ZEA}=H$. zealandica $\mathrm{MEG}=H$. megidis.

matodes, University of California Davis Nematode Collection, Davis, CA, USA. Several slides of paratype hermaphrodites, males, females, together with all stages preserved in TAF, deposited in the Department of Entomology and Nematology, University of Florida, Gainesville, FL, USA.

\section{DIAGNOSIS AND RELATIONSHIPS}

Heterorhabditis safricana n. sp. is characterised by a combination of morphological characters of the hermaphroditic female, male and IJ. The hermaphroditic female can be differentiated from other species by the unique pattern of the vulva. In the male of $H$. safricana n. sp. the gubernaculum is longer than all other species at $24(19-27) \mu \mathrm{m}$. The IJ has a body length of 600 (550676) $\mu \mathrm{m} ; \mathrm{EP}=110(103-122) \mu \mathrm{m}$; tail length (with sheath) $=93(86-108) \mu \mathrm{m}$, and ratio $\mathrm{a}=28.6(24.8$ 31.8).

The IJ of $H$. safricana n. sp. is morphologically closest to $H$. marelatus, $H$. bacteriophora, $H$. downesi, $H$. zealandica and $H$. megidis. It differs from the most closely related species, $H$. marelatus, by shorter average body length $(600 v s 654 \mu \mathrm{m})$ and the shorter average length of the tail (93 vs $107 \mu \mathrm{m}$ ), and from H. bacteriophora in the slightly more posterior position of the excretory pore (110vs $103 \mu \mathrm{m})$, and the slightly longer pharynx (131 vs $125 \mu \mathrm{m})$. 
Males of $H$. safricana $\mathrm{n}$. sp. differ from $H$. marelatus and $H$. downesi in body length 892 (777-1009) vs 942 (805-1046) and 800 (669-876) $\mu \mathrm{m}$, respectively; from $H$. downesi in the more posterior position of the excretory pore at 135 (104-147) vs 89 (86-91) $\mu \mathrm{m}$ from the anterior end and in the length of the pharynx which is 115 (105126) vs 101 (97-106) $\mu \mathrm{m}$ long; from $H$. zealandica in the length of the spicules at 45 (35-54) vs 51 (48-55) $\mu \mathrm{m}$, from $H$. bacteriophora in the length of the pharynx at 115 (105-126) vs 103 (99-105) $\mu \mathrm{m}$ and from H. megidis in shorter body length of 892 (777-1009) vs 1000 (800-1100) $\mu \mathrm{m}$. The new species can be further distinguished from other species by the molecular characteristics of the ITS regions of ribosomal DNA.

\section{BIONOMICS}

The life cycle of this species is similar to that of other species of Heterorhabditis, but IJ of this species can survive and retain infectivity for up to 48 months when stored in filtered water in vented culture flasks at $14^{\circ} \mathrm{C}$, compared to 3-4 months for other Heterorhabditis species.

Infected Galleria turned yellow shortly after death and changed from pink to chocolate brown in the subsequent 10 days while the bacterial paste inside the insect remained yellow.

\section{MOLECULAR ANALYSIS}

\section{DNA characterisation}

The ITS rDNA regions, flanked by primers $18 \mathrm{~S}$ and $26 \mathrm{~S}$, of $H$. safricana $\mathrm{n}$. sp. are characterised by the sequence lengths $(995 \mathrm{bp}$, ITS1 = $379 \mathrm{bp}$, ITS2 = $211 \mathrm{bp}$ ), and nucleotide usage composition (Table 4). Compared to sequences of all other species in the genus, the sequence length of the new species is identical to that of $H$. marelatus but shorter than that of all other species except $H$. baujardi (this sequence is not flanked by primers $18 \mathrm{~S}$ and $26 \mathrm{~S}$ and consequently cannot be compared) (Table 4). The ITS1 sequence length of the new species $(379 \mathrm{bp})$ is the same as that of $H$. marelatus but longer than that of $H$. downesi (374 bp) and $H$. indica (370 bp). It is shorter than that of all others, except $H$. baujardi (unpubl.). The sequence length of the ITS2 region of the new species is $211 \mathrm{bp}$, the same as that of $H$. amazonensis, $H$. baujardi and $H$. marelatus but shorter than that of all other species (Table 4). Genetic distances between the new species, which are presented in Table 5, also can be used to differentiate species in the genus. Heterorhabditis marelatus is the closest species (25 aligned positions) and $H$. floridensis is the most divergent from the new species (168 aligned positions).

\section{PHYLOGENETIC RELATIONSHIPS}

Maximum parsimony (MP) analysis of ITS regions, with gaps treated as missing data, yielded a single most parsimonious tree (tree length $=861, \mathrm{CI}=0.8548$, $\mathrm{HI}=0.2841, \mathrm{RI}=0.8097, \mathrm{RC}=0.6922$; Fig. 5).

Table 4. Sequence length ( $b p=$ base pairs) and composition of ITS regions of ten species of Heterorhabditis.

\begin{tabular}{|c|c|c|c|c|c|c|c|}
\hline $\begin{array}{l}\text { Species } \\
\text { (sequence length) }\end{array}$ & $\begin{array}{l}\text { ITS1 } \\
\text { (bp) }\end{array}$ & $\begin{array}{l}5.8 \mathrm{~S} \\
\text { (bp) }\end{array}$ & $\begin{array}{l}\text { ITS2 } \\
\text { (bp) }\end{array}$ & A & $\mathrm{C}$ & G & $\mathrm{T}$ \\
\hline H. safricana $\mathrm{n} . \mathrm{sp}$. (995 bp) & 379 & 154 & 211 & 0.24121 & 0.21005 & 0.26231 & 0.28643 \\
\hline H. marelatus (995 bp) & 379 & 154 & 211 & 0.23417 & 0.21608 & 0.26432 & 0.28543 \\
\hline H. amazonensis (1010 bp) & 395 & 154 & 211 & 0.25941 & 0.20198 & 0.25545 & 0.28317 \\
\hline H. bacteriophora (1021 bp) & 389 & 154 & 228 & 0.26347 & 0.1998 & 0.25661 & 0.28012 \\
\hline H. baujardi $(795 \mathrm{bp})^{1)}$ & $?$ & 154 & 211 & 0.25732 & 0.19677 & 0.25089 & 0.29502 \\
\hline H. downesi (990 bp) & 374 & 154 & 212 & 0.23838 & 0.21717 & 0.26162 & 0.28283 \\
\hline H. floridensis (1012 bp) & 393 & 154 & 214 & 0.25988 & 0.20059 & 0.25494 & 0.28458 \\
\hline H. indica (988 bp) & 370 & 154 & 215 & 0.26316 & 0.20547 & 0.25506 & 0.27632 \\
\hline H. megidis (1005 bp) & 384 & 154 & 220 & 0.23284 & 0.2199 & 0.27164 & 0.27562 \\
\hline H. mexicana $(1010 \mathrm{bp})$ & 394 & 154 & 213 & 0.25545 & 0.20198 & 0.2604 & 0.28218 \\
\hline H. zealandica (1003 bp) & 387 & 154 & 212 & 0.22233 & 0.21834 & 0.27318 & 0.28614 \\
\hline
\end{tabular}

1) This sequence is not as complete as in other species.

? Not available. 
Table 5. Pairwise distances between taxa. Below diagonal: Total character differences; above diagonal: Mean character differences (adjusted for missing data).

\begin{tabular}{|c|c|c|c|c|c|c|c|c|c|c|c|c|c|}
\hline & & 1 & 2 & 3 & 4 & 5 & 6 & 7 & 8 & 9 & 10 & 11 & 12 \\
\hline 1 & H. safricana $\mathrm{n} . \mathrm{sp}$. & - & 0.0252 & 0.0324 & 0.0558 & 0.0909 & 0.1273 & 0.1 & 0.2 & 0.16 & 0.1 & 0 . & 0.4191 \\
\hline 2 & H. marelatus & 25 & - & 0.0374 & 0.0589 & 0.0860 & 0.12 & 0.1564 & 0.2056 & 0.1607 & 0.1687 & 0.1706 & 0.4160 \\
\hline 3 & H. downesi & 32 & 37 & - & 0.0396 & 0.0761 & 0.1259 & 0.1550 & 0.2059 & 0.1596 & 0.1677 & 0.1696 & 0.4134 \\
\hline 4 & H. megidis & 55 & 58 & 39 & - & 0.0996 & 0.1405 & 0.1714 & 0.2225 & 0.1737 & 0.1787 & 0.1806 & 0.4244 \\
\hline 5 & H. zealandica & 90 & 85 & 75 & 98 & - & 0.1592 & 0.1835 & 0.2358 & 0.1885 & 0.1965 & 0.1973 & 0.4226 \\
\hline 6 & H. bacteriophora & 125 & 123 & 123 & 137 & 157 & - & 0.1533 & 0.2150 & 0.1692 & 0.1773 & 0.17 & 0.4322 \\
\hline 7 & H. indica & 147 & 150 & 148 & 163 & 176 & 147 & - & 0.0946 & 0.1692 & 0.0786 & 0.0796 & 0.4288 \\
\hline 8 & H. baujardi & 157 & 155 & 154 & 168 & 179 & 163 & 72 & - & 0.0189 & 0.0303 & 0.0240 & 0.4897 \\
\hline 9 & H. amazonensis & 158 & 156 & 154 & 168 & 184 & 165 & 67 & 15 & - & 0.0218 & 0.0198 & 0.4404 \\
\hline 10 & H. mexicana & 166 & 164 & 162 & 173 & 192 & 173 & 7 & 24 & 22 & - & 0.0158 & 0.4379 \\
\hline 11 & H. floridensis & 168 & 166 & 164 & 175 & 193 & 175 & 78 & 19 & 20 & 16 & - & 0.4415 \\
\hline 12 & C. elegans & 409 & 406 & 401 & 418 & 415 & 430 & 416 & 380 & 436 & 434 & 438 & - \\
\hline
\end{tabular}

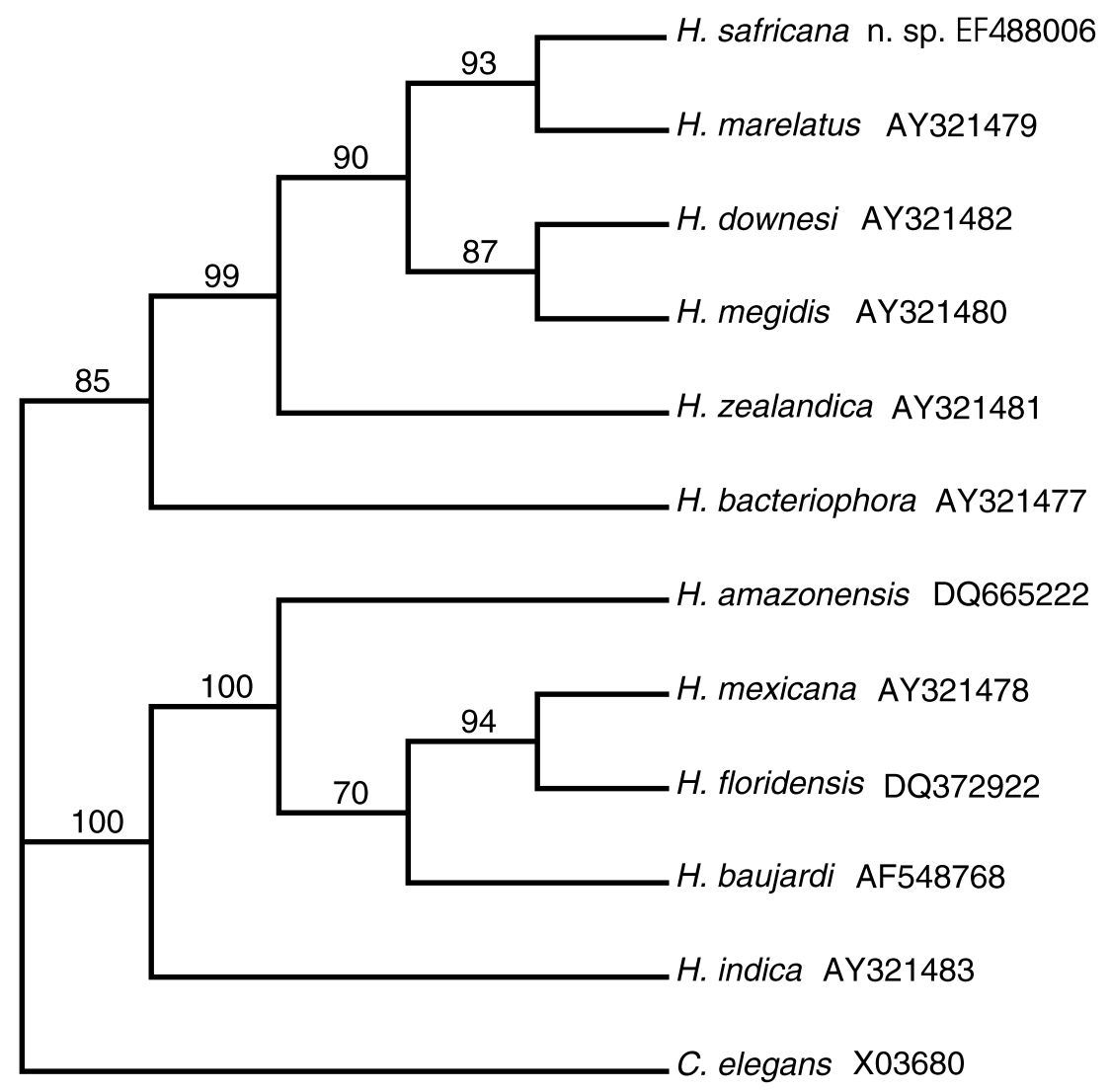

Fig. 5. Phylogenetic relationships of 11 species of Heterorhabditis based on analysis of ITS $r$ DNA regions. Maximum parsimony produced two monophyletic groups, the megidis-group $(\mathrm{H}$. bacteriophora, H. downesi, H. marelatus, H. megidis, H. safricana $n$. $s p$. and $\mathrm{H}$. zealandica) and the indica-group (H. amazonensis, H. baujardi, H. floridensis, H. indica and H. mexicana). Heterorhabditis bacteriophora clustered with the megidis-group with a weaker bootstrap support. Numbers at the nodes represent bootstrap proportions (50\% or more, 100 replicates). 
With gaps treated as a fifth base, MP analysis resulted in a tree (tree length $=1366, \mathrm{CI}=0.8668, \mathrm{HI}=0.1332$, $\mathrm{RI}=0.8047, \mathrm{RC}=0.6975)$ with a topology congruent with the aforementioned tree. The parsimonious tree included two monophyletic groups. In the first, the megidis-group, $H$. safricana n. sp., H. downesi, H. marelatus and $H$. megidis comprise a monophyletic group well supported by bootstrap analysis (90\%). In the megidisgroup, $H$. safricana n. sp. and H. marelatus formed one of two monophyletic sister groups with $H$. downesi and $H$. megidis comprising the other; $H$. zealandica was sister taxon to the group formed by the other four. Heterorhabditis bacteriophora clustered with the megidis-group with a weaker bootstrap support (85\%). If new, closely related species are described, $H$. bacteriophora will probably form a separate group. The second group, the indicagroup was formed by six other species well supported by bootstrap analysis (100\%). Maximum likelihood analysis of ITS regions generated a tree (Fig. 6) with a topology somewhat different from that of the MP tree. Bootstrap indices were weak for some nodes. The trees yielded by using hLRT and AIC were identical in topology with just a slight difference in bootstrap support (Fig. 6). The reconstructed nucleotide character transformations (Fig. 7) showed that the new species differs from its closest taxon, $H$. marelatus, at 25 aligned positions, seven of which are

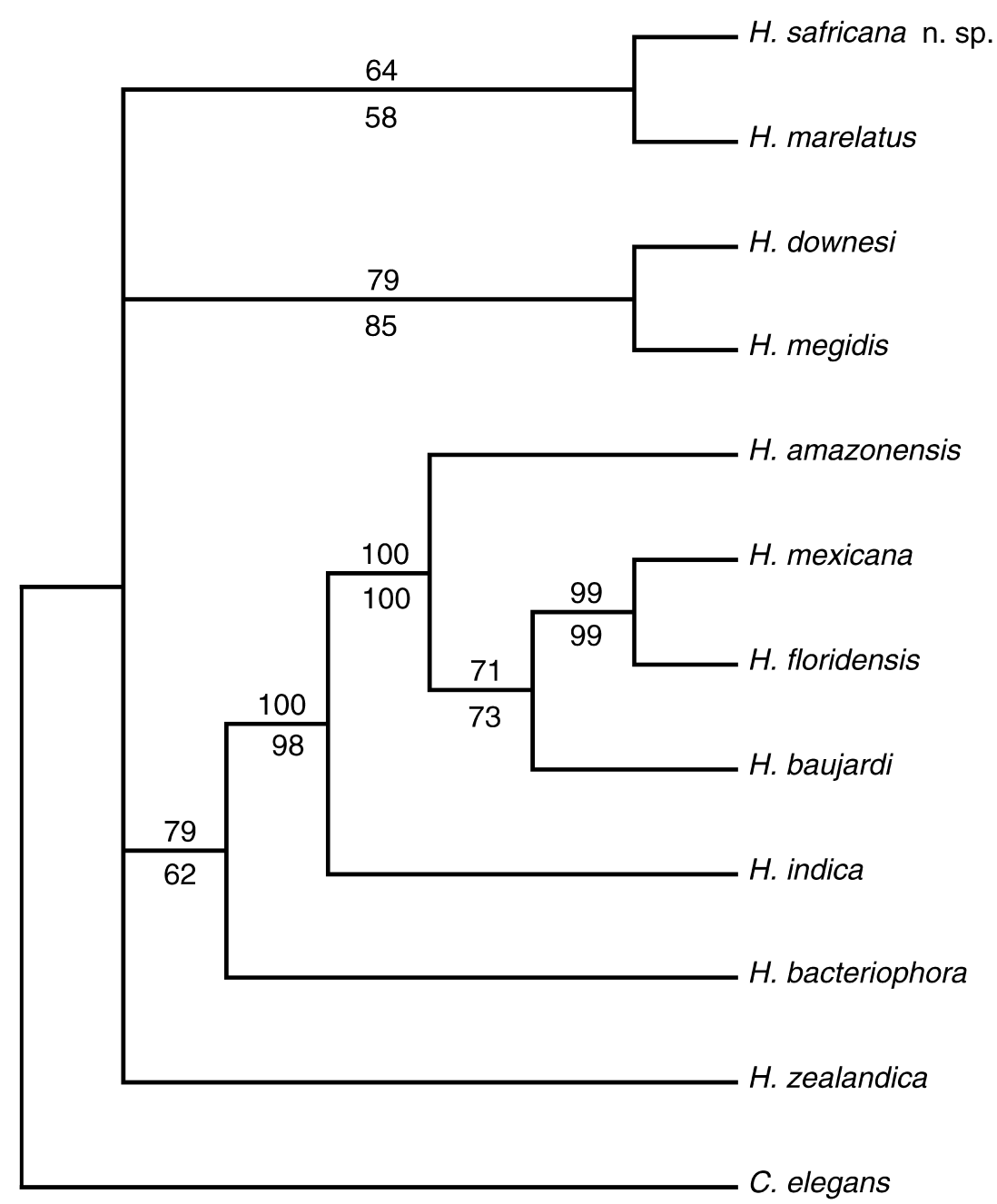

Fig. 6. Phylogenetic relationships of 11 species of Heterorhabditis using maximum likelihood analysis of the ITS regions. Numbers at the nodes represent bootstrap proportions, numbers above and below branches are from the analysis using hLRT and AIC, respectively (50\% or more, 100 replicates). Accession numbers are cited in Figure 5. 


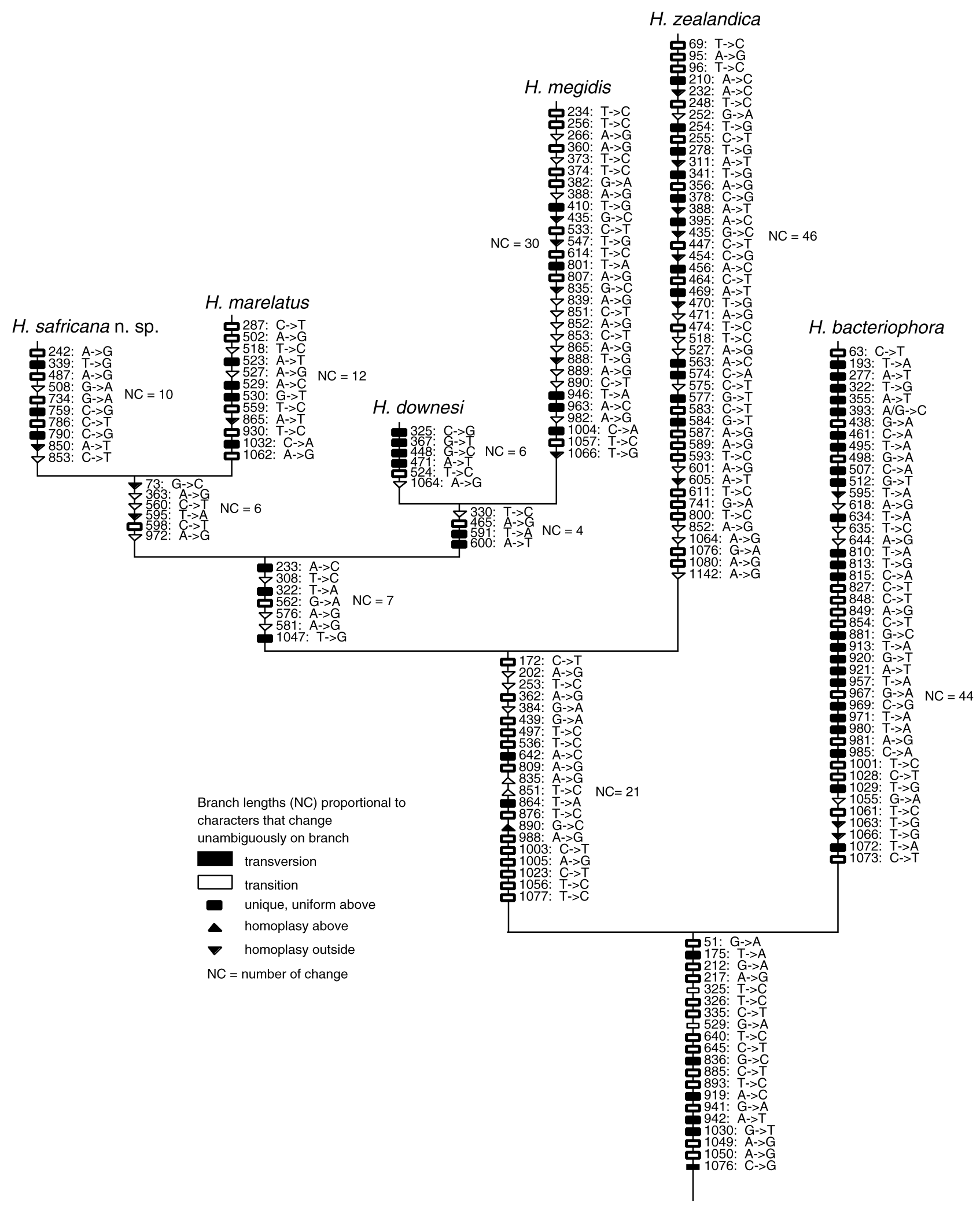


Fig. 7. Clade containing Heterorhabditis safricana n. sp. and nearest neighbours in the megidis-group (H. safricana, H. marelatus, H. downesi, H. megidis, H. zealandica and H. bacteriophora). Phylogeny reconstructed from ITS rDNA sequences (maximum parsimony). Mapped character states (unambiguous changes only; MacClade 4.05) identify character support, number of change and autapomorphies (= unique, derived characters; boxes at the position 242, 339, 487, 743, 759, 786 and 790 of the transformation series), four are transitions and three are transversions.

unambiguous, polarised autapomorphies (three transversions and four transitions) and the number of characters that changed unambiguously (NC) on the branch is 10 . For the closest species, $H$. marelatus, the new species has nine autapomorphies and $\mathrm{NC}=12$.

We conclude that the morphological and molecular characteristics that we found are sufficient to conclude that $H$. safricana is a new species.

\section{Acknowledgements}

The authors thank the Deciduous Fruit Producers Trust for funding the project and Nemlab for providing soil samples.

\section{References}

Andaló, V., Nguyen, K.B. \& Moino JR, A. (2006). Heterorhabditis amazonensis $\mathrm{n}$. sp. (Rhabditida: Heterorhabditidae) from Amazonas, Brazil. Nematology 8, 853-867.

BedDing, R.A. \& AKHURST, R.J. (1975). A simple technique for the detection of insect parasitic rhabditid nematodes in soil. Nematologica 21, 109-110.

Courtney, W.D., Polley, D. \& Miller, V.I. (1955). TAF, an improved fixative in nematode technique. Plant Disease Reporter 39, 570-571.

DutKy, S.R., Thompson, J.V. \& CANTwell, G.E. (1964). A technique for the mass propagation of the DD-136 nematode. Journal of Insect Pathology 6, 417-422.

Grenier, E., Bionifassi, E., Abad, P. \& Laumond, D. (1996). Use of species specific satellite DNAs as diagnostic probes in the identification of Steinernematidae and Heterorhabditidae entomopathogenic nematodes. Parasitology 113, 483-489.

HARINGTON, J.S. (1953). Observation on the biology, the parasites and the taxonomic position of the maize beetle Heteronychus sanhelenae Blanch. South African Journal of Science 50, 11-14.

KLEIN, M.G. (1990). Efficacy against soil-inhibiting insect pests. In: Gaugler, R. \& Kaya, H.K. (Eds). Entomopathogenic nematodes in biological control. Boca Raton, FL, USA, CRC Press, pp. 195-214.
LiU, J. \& BERRY, R.E. (1996). Heterorhabditis marelatus $\mathrm{n}$. sp. (Rhabditida: Heterorhabditidae) from Oregon. Journal of Invertebrate Pathology 67, 48-54.

Malan, A.P., NGuYen, K.B. \& Addison, M.F. (2006). Entomopathogenic nematodes (Steinernematidae and Heterorhabditidae) from the southwestern parts of South Africa. African Plant Protection 12, 65-69.

NGUYen, K.B. (2006). Morphology and taxonomy of entomopathogenic nematodes. Available online from http://kbn.ifas.ufl.edu/kbnstein.htm (accessed April 20, 2006).

NGuYen, K.B. \& SMART JR, G.C. (1995a). Morphometrics of infective juveniles of Steinernema spp. and Heterorhabditis bacteriophora (Nematoda: Rhabditida). Journal of Nematology 27, 206-212.

Nguyen, K.B. \& SMART JR, G.C. (1995b). Scanning electron microscope studies of Steinernema glaseri (Nematoda: Steinernematidae). Nematologica 41, 183-190.

Nguyen, K.B., Shapiro-Ilan, D.I., Stuart, R.J., MCCoY, D.W., JAMES, R.R. \& ADAMS, B.J. (2004). Heterorhabditis mexicana n. sp. (Rhabditida: Heterorhabditidae) from Tamaulipas, Mexico, and morphological studies of the bursa of Heterorhabditis spp. Nematology 6, 321-244.

Nguyen, K.B., Gozel, U., Koppenhöfer, H.S. \& ADAMS, B.J. (2006a). Heterorhabditis floridensis n. sp. (Rhabditida: Heterorhabditidae) from Florida. Zootaxa 1177, $1-19$.

Nguyen, K.B., Malan, A.P. \& Gozel, U. (2006b). Steinernema khoisanae n. sp. (Rhabditida: Steinernematidae), a new entomopathogenic nematode from South Africa. Nematology 8, 157-175.

Phan, K.L., Subbotin, S.A., Nguyen, N.C. \& Moens, M. (2003). Heterorhabditis baujardi sp. n. (Rhabditida: Heterorhabditidae) from Vietnam and morphometric data for H. indica populations. Nematology 5, 367-382.

PoINAR JR, G.O. (1976). Description and biology of a new insect parasitic rhabditoid, Heterorhabditis bacteriophora $\mathrm{n}$. gen., n. sp. (Rhabditida; Heterorhabditidae n. fam.). Nematologica 21 (1975), 463-470.

POINAR JR, G.O. (1990). Taxonomy and biology of Steinernematidae and Heterorhabditidae. In: Gaugler, R. \& Kaya, H.K. (Eds). Entomopathgenic nematodes in biological control. Boca Raton, FL, USA, CRC Press, pp. 23-60.

PoinAR JR, G.O., JACKSON, T. \& KLEIN, M. (1987). Heterorhabditis megidis sp. n. (Heterorhabditidae: Rhabditida) parasitic in the Japanese beetle, Popillia japonica (Scarabaei- 
dae: Coleoptera), in Ohio. Proceedings of the Helminthological Society of Washington 54, 53-59.

POINAR JR, G.O., KARUNAKAR, G.D. \& DAVID, H. (1992). Heterorhabditis indica n. sp. (Rhabditida: Nematoda) from India: separation of Heterorhabditis spp. by infective juveniles. Fundamental and Applied Nematology 15, 467-472.

PosAdA, D. \& CRANDALL, K.A. (1998). MODELTEST: testing the model of DNA substitution. Bioinformatics 14, 817-818.

SEINHORST, J.W. (1959). A rapid method for the transfer of nematodes from fixative to anhydrous glycerin. Nematologica 4, 67-69.

Shapiro-Ilan, D.I., Gouge, D.J. \& Koppenhöfer, A.M. (2002). Factors affecting commercial success: case studies in cotton, turf and citrus. In: Gaugler, R. (Ed.). Entomopathogenic nematology. Wallingford, UK, CABI Publishing, pp. 333-356.

SPAULL, V.W. (1988). A preliminary evaluation of entomogenous nematodes to control the African sugarcane stalk borer Eldana saccharina (Lepidoptera: Pyralidae). Proceedings of the South African Sugar Technologists' Association 62, 120123.

SPaull, V.W. (1990). Field tests to control the pyralid, Eldana saccharina, with an entomogenous nematode, Heterorhabditis sp. Proceedings of the South African Sugar Technologists' Association 64, 103-106.

SPAULl, V.W. (1991). Heterorhabditis and Steinernema species (Nematoda: Rhabditida) for the control of a sugar cane stalk borer in South Africa. Phytophylactica 23, 213-215.

Stock, S.P., Griffin, C.T. \& BURNELL, A.M. (2002). Morphological characterization of three isolates of Heterorhabditis Poinar, 1976 from the "Irish group" (Nematoda: Rhabditida: Heterorhabditidae) and additional evidence supporting their recognition as a distinct species, $H$. downesi n. sp. Systematic Parasitology 51, 95-106.

SWOFFORD, D.L. (2002). PAUP* Phylogenetic Analysis Using Parsimony (and other methods) 4.0 beta. Sunderland, MA, USA, Sinauer Associates.

WoOdRING, J.L. \& KAYA, H.K. (1988). Steinernematid and heterorhabditid nematodes: A handbook of techniques. Southern Cooperative Series Bulletin 331, pp. 1-30. 